\title{
Brief psychodynamic interpersonal psychotherapy for patients with multisomatoform disorder: randomised controlled trial
}

\author{
H. Sattel, C. Lahmann, H. Gündel, E. Guthrie, J. Kruse, M. Noll-Hussong, C. Ohmann, J. Ronel, \\ M. Sack, N. Sauer, G. Schneider and P. Henningsen
}

\section{Background}

Multisomatoform disorder is characterised by severe and disabling bodily symptoms, and pain is one of the most common and impairing of these. Furthermore, these bodily symptoms cannot be explained by an underlying organic disorder. Patients with multisomatoform disorder are commonly found at all levels of healthcare and are typically difficult to treat for physicians as well as for mental health specialists

\section{Aims \\ To test whether brief psychodynamic interpersonal therapy (PIT) effectively improves the physical quality of life in patients who have had multisomatoform disorder for at least 2 years.}

\section{Method}

We recruited 211 patients (from six German academic outpatient centres) who met the criteria for multisomatoform disorder for a randomised, controlled, 12-week, parallelgroup trial from 1 July 2006 to 1 January 2009 (International Standard Randomised Controlled Trial Number ISRCTN23215121). We randomly assigned the patients to receive either 12 weekly sessions of PIT $(n=107)$ or three sessions of enhanced medical care $(E M C, n=104)$. The physical component summary of the Short Form Health Survey (SF-36) was the pre-specified primary outcome at a 9-month follow-up.

\section{Results}

Psychodynamic interpersonal therapy improved patients' physical quality of life at follow-up better than EMC (mean improvement in SF-36 score: PIT 5.3, EMC 2.2), with a small to medium between-group effect size $(d=0.42$, $95 \% \mathrm{Cl} 0.15-0.69, P=0.001)$. We also observed a significant improvement in somatisation but not in depression, health anxiety or healthcare utilisation.

\section{Conclusions}

This trial documents the long-term efficacy of brief PIT for improving the physical quality of life in patients with multiple, difficult-to-treat, medically unexplained symptoms.

\section{Declaration of interest}

None.
Patients with severe and disabling medically unexplained symptoms are common in the healthcare system. Many of these patients are heavy users of healthcare and incur large costs to health services and society. Moreover, hospital clinicians, primary care physicians and mental health specialists often find these patients difficult to treat. ${ }^{1,2}$ Medically unexplained symptoms are classified as somatoform disorders, a DSM-IV mental health category, and in parallel as functional somatic syndromes such as irritable bowel syndrome or fibromyalgia that are typically within the realms of general and specialist medicine. ${ }^{3,4}$ All of these disorders are characterised by distressing somatic symptoms that significantly disrupt daily life. Pain is one of the most frequent and clinically relevant complaints alongside functional complaints such as diarrhoea, dizziness and fatigue. ${ }^{5-7}$ Evidence has accumulated showing that psychotherapy is a promising option for these disorders. ${ }^{2}$ Both cognitive-behavioural therapy (CBT) and short-term psychodynamic therapies show at least some efficacy. ${ }^{8-10}$ Thus far, however, trials have been of less than adequate size or limited to one functional somatic syndrome. Therefore, the existing data do not cover the clinical reality of patients with complex, overlapping diagnoses. ${ }^{2,8-10}$

Against this background we conducted a large, multicentre, randomised controlled trial to test the efficacy of a manualised brief psychodynamic interpersonal psychotherapy (PIT), a variant of psychodynamic therapy with particular emphasis on interpersonal processes, ${ }^{11}$ not limited to one specific functional somatic syndrome. We used the diagnostic construct of multisomatoform disorder originally suggested by Kroenke et al as a common point of reference for patients recruited to the trial from different somatic specialties. ${ }^{12}$ This construct characterises a medium-severe form of the disorder. As pain is the most frequent complaint in patients with this disorder we chose pain irrespective of its location as the presenting symptom to reduce the heterogeneity of the patient group, to ensure that our sample was clinically relevant and to serve as a lynchpin for symptomoriented therapeutic goals. As recorded in the International Standard Randomised Controlled Trial Number trial registration (ISRCTN23215121), our predefined hypothesis was that PIT would prove to be efficacious compared with enhanced medical care, with physical quality of life at follow-up as the primary outcome, whereas other clinical features of the targeted disorder (e.g. depression, health anxiety or healthcare use) would not necessarily be affected.

\section{Method}

We conducted this study at six university departments of psychosomatic medicine in Germany (Munich, Düsseldorf, Hannover, Heidelberg, Münster and Regensburg). We recruited patients from the out-patient departments of neurology and internal medicine as well as from pain treatment centres and an orthopaedics private practice. The independent clinical trials unit at the University of Düsseldorf stored all the data, regularly monitored all project sites and analysed the primary and secondary outcome data. The ethics committees at all sites approved the study. 


\section{Participants}

Between June 2006 and December 2007 we used the 15-item Patient Health Questionnaire (PHQ-15) and the 36-item Short Form Health Survey (SF-36) to screen consecutive patients, aged 18-77 years, who presented at somatic out-patient departments. ${ }^{13,14}$ Trained psychologists and physicians from the respective departments of psychosomatic medicine interviewed the patients with possible multisomatoform disorder (PHQ-15 scores $>10$ ) and markedly impaired physical quality of life (SF-36 scores <40) using the somatoform disorders and hypochondria sections of the Structured Clinical Interview for DSM-IV (SCID). ${ }^{15}$ We modified the interview to check for the presence of multisomatoform disorder according to the published criteria. ${ }^{12}$ A multisomatoform disorder diagnosis requires a minimum of three current somatoform symptoms (pain, dizziness, bowel dysfunction, fatigue, etc.) that are functionally disabling and that an organic disease or another mental disorder cannot sufficiently explain, and a history of somatoform symptoms on at least half of the days over at least 2 years, resulting in healthcare use. ${ }^{12}$ We reviewed the medical charts and contacted the treating physicians to rule out possible or unclear organic explanations for the symptoms.

Patients were eligible for trial inclusion if they fulfilled the criteria for multisomatoform disorder and had at least one pain-related symptom as well as an SF-36 physical component summary (PCS) score of 40 or less. We selected only those patients with significant impairments of physical quality of life (according to the proposed DSM- 5 criteria) ${ }^{8}$ to ensure that the results of these interventions could be compared regarding their differential effect on this central clinical feature. We excluded patients with insufficient cognitive abilities (a Mini-Mental State Examination score $<27),{ }^{16}$ severe, chronic and disabling somatic disease or severe comorbid mental disorder that caused major impairment in social functioning (e.g. schizophrenia, severe forms of bipolar disorder or substance misuse), as well as those who were at risk of suicide, were undergoing psychotherapy at the time of the screening or could not speak German. Additionally, we excluded a small number of patients with a DSM-IV diagnosis of hypochondriasis ( $n=5$ in the screened sample) because its main feature consists of excessive worry about illness and the intractable belief that one has an undiagnosed physical disease; patients with somatoform disorders, on the other hand, are impaired primarily by their physical symptoms. Patients with hypochondriasis require a focus on health anxiety, for which effective treatments are available, such as $\mathrm{CBT}^{17}$ We did not exclude patients with major depression or an anxiety disorder if there was no major clinically detectable impairment in social functioning.

\section{Randomisation and assessment}

We obtained written informed consent after fully explaining the study to the participants. A computer program generated a blocked randomisation list and the Coordination Centre for Clinical Trials covertly applied this list to our sample. Specifically, the centre applied stratified random blocks of four, six or eight patients. After receiving informed consent, we submitted a randomisation request and the centre returned the result for the patient in question within $24 \mathrm{~h}$. The patients completed the baseline assessment independently immediately after providing informed consent and delivered the assessment to the study therapist in person or by post. Thereafter, the therapist disclosed the group assignment to the patient and treatment began. When asked, $28 \%$ of the patients assigned to the EMC group disapproved of their assignment, but this opinion was not associated with study attrition.
The assessment questionnaires at the end of the therapy and for the 9-month follow-up period were handed out by the therapist after the last session or sent by post shortly afterwards. The patients completed the questionnaires independently and returned them, usually by post.

\section{Interventions}

The experimental intervention consisted of 12 weekly sessions of PIT specifically adapted to the needs of patients in bodily distress. The first session lasted up to $90 \mathrm{~min}$; all other sessions were approximately $45 \mathrm{~min}$ long. Conceptually, this interpersonal variant of psychodynamic therapy is similar to modern variants such as mentalisation-based therapy, in that it assumes that developmentally based dysregulations of (bodily) self-experience and relationships rather than unconscious conflicts are the primary basis for the symptoms. ${ }^{18,19}$ The PIT treatment manual was developed jointly and pilot-tested over the course of 3 years. ${ }^{20}$ It describes three phases. In the first phase the emphasis lies on building a therapeutic relationship by underscoring the legitimacy of bodily complaints. This relationship is achieved by assessing bodily complaints, clarifying the associated disappointments and other negative experiences with the healthcare system and introducing the possibility that there are links between emotional states and bodily complaints. In addition, bodily relaxation training is introduced and preferably maintained during the three phases of therapy. Finally, the patients are educated about the emergence of their symptoms in an appropriate and noncatastrophising manner. These basic measures are comparable to those applied in currently approved CBT approaches. ${ }^{21}$

The second phase of PIT (consisting of at least six sessions) focuses on clarifying the patient's emotions as they relate to symptoms within the person's own life. To this end, the links between bodily complaints and emotions that were introduced in the first phase are explored in greater detail. Furthermore, these links are discussed in the context of actual relationships. Associations are also established between the experience of severe bodily distress and earlier relationships. This goal is based on the assumption that early dysfunctional or traumatic bodily relationship experiences shape the tendency to experience disabling bodily symptoms that the patient cannot explain or control later in life. ${ }^{20}$ Identifying these dysfunctional patterns is assumed to be essential for improving the regulation of bodily self-experience and symptoms. The third phase of the therapy concentrates on termination issues, such as taking stock and planning additional psychological, social or pharmacological therapeutic measures for well-defined psychological symptoms or bodily symptoms without medical explanation. Finally, the patients move to the post-treatment phase.

After recruitment we treated the participants in the outpatient departments of psychosomatic medicine. The therapists, four psychologists and four physicians with at least 3 years of training in psychotherapy, who were employed at these departments, were trained centrally and jointly in Heidelberg and Munich in the use of the manual by the group who developed the manual (P.H., H.G., E.G., J.K., C.L., N.S. and G.S.). The local principal investigators supervised all the psychotherapies. We recorded all the sessions using video- or audiotape. Based on these recordings, we used a modified version of the Sheffield Psychotherapy Rating Scale to rate adherence to the experimental intervention. $^{22}$ We rated the non-specific therapeutic and PIT interventions according to their frequency and intensity. We also included an additional 13 items to cover disorder-specific conditions relevant to treating multisomatoform disorder. The interrater reliability (Cronbach's $\alpha$ ) for this modified scale was 
0.68. We tested adherence to the intervention in 45 randomly selected PIT therapy sessions from the second phase, which - in contrast to the highly structured first and final phases - focused on interpersonal issues. On a seven-point scale for typical PIT interventions, ranging from 1 (not present) to 7 (extensively present), we rated 3 of the 13 major therapeutic elements with at least a 5 (considerably observable/present) in $87 \%$ of sessions.

The EMC comparison group received education and counselling regarding the therapeutic alternatives based on the evidence-based guidelines for the treatment of somatoform disorders/functional somatic syndromes in primary and somatic specialist care. ${ }^{23}$ Instead of telling the patients that their symptoms had no physical cause, the therapists discussed an explanatory model of the development and maintenance of their symptoms. This model included advice on symptom management, specific pharmacological or psychotherapeutic treatments for either welldefined psychological symptoms or pain and healthcare use (e.g. avoiding unnecessary somatic diagnostic and/ or therapeutic interventions). The patients had three approximately half-hour sessions at 6-week intervals with physicians specifically trained in EMC (also based on a manual) at their respective departments of psychosomatic medicine. Nine specialists at the referring outpatient department provided EMC. The therapists controlled treatment adherence using checklists and covered $94 \%$ of all the assessed elements in at least one session. ${ }^{18}$ We considered this type of medical care to be 'enhanced', as (unlike typical clinical practice) it strictly observed recommended national guidelines.

During the treatment phase we told the participants not to initiate additional treatments and to report primary or specialist care contacts. At the end of therapy the clinicians recommended additional psychotherapeutic or somatic treatments and medication for the patients in a comparable manner to the PIT intervention.

\section{Measures}

We assessed the patients at baseline $\left(t_{1}\right)$, at the end of treatment ( 3 months after baseline, $t_{2}$ ) and - to evaluate the long-term therapeutic effects - at a follow-up assessment 9 months after the end of treatment $\left(t_{3}\right){ }^{9}$ As the outcome assessment consisted of self-report measures, masking procedures were not applicable. All the measures were delivered in their validated German versions. In keeping with the current debates regarding treatment success in functional somatic syndromes, a lesser degree of symptom impairment or improvements in functional bodily health (as opposed to complete relief of symptoms) often already indicate an effective treatment. ${ }^{2,12}$ Therefore, we felt that it was important to use a health-related quality of life measure as the primary outcome, and selected the improvement in the physical component summary (range $0-100$ ) of the SF-36, ${ }^{14}$ as measured at $t_{3}$. The PCS has been shown to be a valid and change-sensitive indicator of bodily functioning and quality of life; ${ }^{24}$ moreover, it addresses the major concerns of the patients included in our trial more directly than the mental component summary. Patients regarded PCS changes of $2.5,4.5$ and 6.5 points respectively as clinically small, moderate and large. ${ }^{25}$ In a previous PIT trial investigating patients with a functional somatic syndrome, ${ }^{26}$ those who received PIT improved by a mean of 5.5 points on the PCS. The median number of days of restricted activity per month was also reduced from 5 to 0 . As a consequence, we determined that a mean increase of 5 points (corresponding to an effect size of 0.5) in an individual patient would indicate a clinically significant change. This increase could result from a moderate improvement in six areas of physical functioning or a moderate reduction in both pain and pain-related disability.
As the sustainability of potential treatment effects is particularly important in a chronic condition such as multisomatoform disorder, we chose improvement at $t_{3}$ rather than $t_{2}$ as the primary end-point. This decision was in keeping with the observation that the effects of psychotherapy often increase after the end of the treatment. ${ }^{9,27}$ The secondary outcome measures assessed aspects of psychopathology or potential mediators of change. The measures reported here included the MCS (range 0-100) of the SF-36; the Patient Health Questionnaire, which contains a somatisation module (PHQ-15, range 0-30) as well as a depression module (PHQ-9, range $0-27){ }^{13}$ and the Whitely Index Short Form (WI-7) to assess health anxiety. ${ }^{28,29}$ The questions on the WI-7 (range 0-7) refer to the preceding 4 weeks, and a cut-off score of 4 or more points indicates clinically relevant health anxiety. We recorded the number of times a patient visited either a primary care physician or specialist, the use of antidepressants and the use of psychotherapy in the 3 months preceding each time point (except for other psychotherapy at $t_{2}$, as this was an exclusion criteria) to assess aspects of healthcare use.

\section{Statistical analysis}

Analyses, under the direction of the independent clinical trials unit at the University of Düsseldorf, were based on the intentto-treat method for all participants and confirmed that the missing data for the primary and secondary outcomes were missing at random. This assumption holds when the missing data can be traced back to the observed data and no study-specific reason can account for their absence. Multiple imputations were used to replace missing data, which consisted mainly of patients who were lost to follow-up $(n=26)$. We performed sensitivity analyses to estimate the potential differences in the imputed outcomes $v$. those derived from incomplete data. A sample size of 110 patients per group combined with an expected attrition rate of $20 \%$ provided $80 \%$ power to detect a moderate treatment effect size of 0.5. We compared the $t_{1}$ clinical and demographic variables of the two treatment groups using chi-squared tests for dichotomous or nominally distributed variables and $t$-tests for continuous variables.

We tested the treatment effects in both groups across $t_{1}, t_{2}$ and $t_{3}$ using linear mixed models (LMM) by analysing the differences between baseline and post-treatment or 9-month follow-up respectively and controlling for study site, age, gender and education. We report the confidence intervals for the differences between the groups. We analysed the secondary outcomes and clinical characteristics such as depression and anxiety in a similar manner. To control for differences at $t_{1}$ we included the initial number of primary care and specialist visits in the LMM. We compared changes in antidepressant and psychotherapy use between the groups using Mann-Whitney $U$-tests. The Bonferroni correction for multiple tests was used and resulted in adjusting the alpha level for significance to $0.01(0.05 / 5)$. We performed the statistical analyses using SPSS for Windows version 18.0. The reporting of this trial follows the Consort criteria. ${ }^{30}$

\section{Results}

In total 211 participants gave informed consent and were randomised to PIT $(n=107)$ or EMC $(n=104)$; three withdrew consent to participate immediately after randomisation (Fig. 1). The withdrawal rates were $19 \%$ for PIT and $27 \%$ for EMC. Respectively, $10 \%$ and $15 \%$ of the patients in the PIT and EMC groups did not provide data at $t_{3}$. The attrition rates 


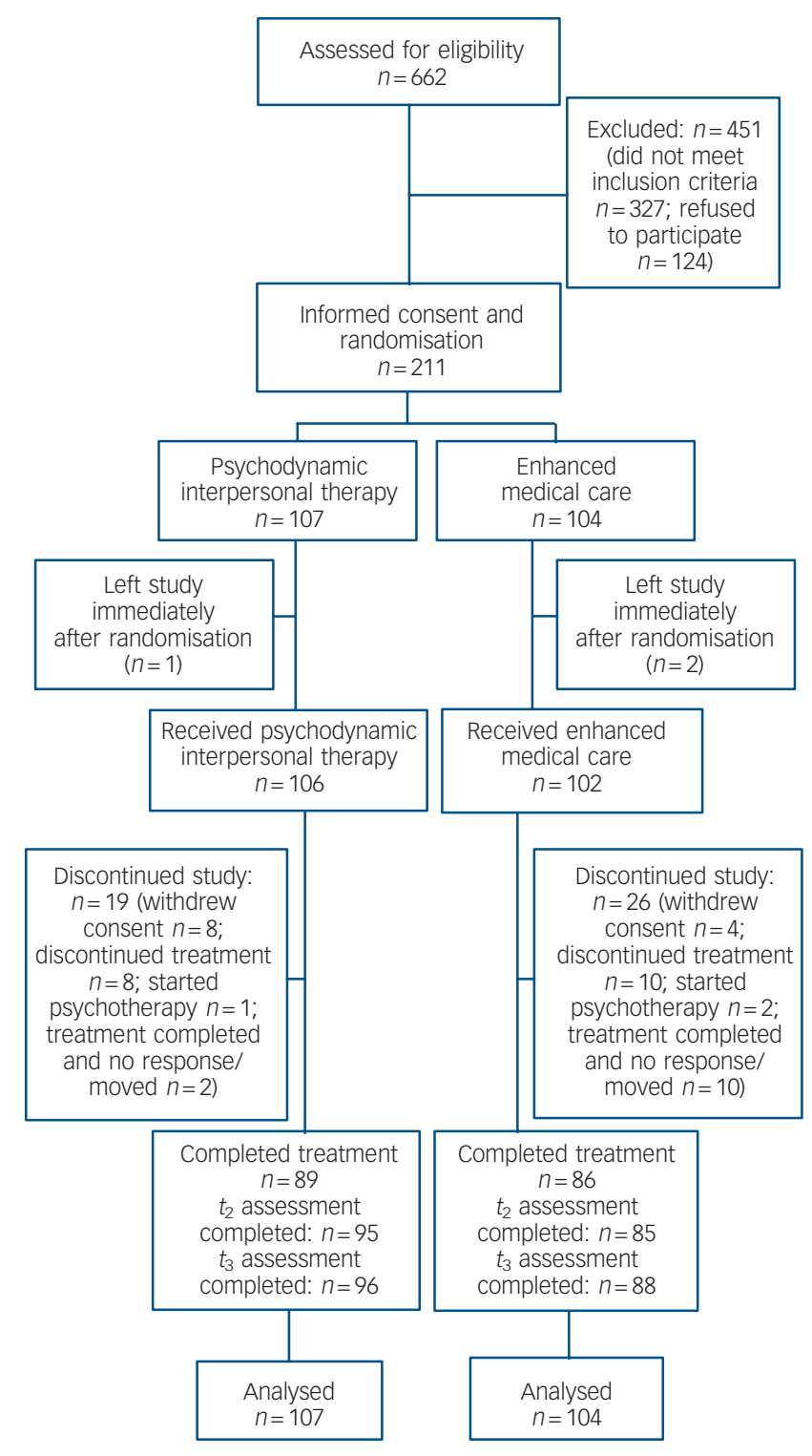

Fig. 1 Study profile ( $t_{2}$, end of therapy; $t_{3}$, 9-month follow-up).

did not significantly differ between the groups. Nine patients who received PIT and twelve EMC group patients who did not complete the treatment sent back the questionnaires at the 9-month follow-up. These patients demonstrated comparable improvements in PCS score of 3.2 (PIT) and 3.8 points (EMC). Two patients were admitted to a mental hospital for a short period owing to suicidal thoughts during the treatment phase (one in PIT and one in EMC). However, both patients eventually continued with their treatments.

\section{Characteristics at $t_{1}$}

At baseline we found no significant difference in age, gender, marital status, education or employment status between the two study groups (Table 1). Patients in both groups were chronically ill with multiple symptoms; on average they had more than ten somatoform symptoms (according to the SCID). The mean duration of these symptoms was 10 years. The only characteristic difference between the participating sites was participant age; in particular, differences in the treatment effect between the sites proved to be negligible. At $t_{1}, 44 \%$ and $39 \%$ of the patients in the PIT and EMC groups respectively fulfilled the DSM-IV criteria for major depression according to the PHQ-9 (i.e. having at least five depressive symptoms, at least one of which was depressed mood or loss of interest or pleasure, over a 2-week period and having these symptoms represent a change from previous functioning). Furthermore, $49 \%$ and $45 \%$ of the patients in the PIT and EMC groups respectively received antidepressants, which were stopped for both groups when their depressive symptoms improved. Using the cut-off of 4 points on the WI-7 scale the majority of patients in both groups were significantly impaired by clinically relevant health anxiety $(55 \%$ in the PIT group and 56\% in the EMC group).

\section{Clinical outcomes}

Table 2 shows the primary and secondary outcomes for PIT and EMC groups at the three assessment points. In terms of the primary outcome - physical quality of life as measured by the SF-36 PCS at $t_{3}$ - patients in both groups improved from $t_{1}$. However, the improvement of the patients in the PIT group was significantly greater than that of the EMC group, according to the LMM analysis and in tests of actual group differences at $t_{3}$. The mean increase for the PIT group was 5.3 points, which passed the level of clinical significance that was set before we collected the data. Specifically, $43 \%$ of the PIT group who received psychotherapy reported 5 or more points of improvement at $t_{3}$ (26\% for the EMC patients; odds ratio 2.13, 95\% CI 1.19-3.80). The between-group effect size $(d=0.42)$ was moderate. The difference between the groups became apparent in the period between $t_{2}$ and $t_{3}$, in which the PCS ratings of the PIT group increased further and those of the EMC group decreased slightly.

We also observed treatment effects from $t_{1}$ to $t_{3}$ for both groups in the patients' secondary outcomes. Health anxiety improved in both groups; in addition, the PIT group improved their PHQ-15 somatisation and PHQ-9 depression scores, and the EMC group improved their MCS scores. We observed differential treatment effects between the groups for the PHQ-15 somatisation score, which was significantly improved in the PIT group 9 months after the end of the treatment. Despite the rate of major depressive syndrome significantly decreasing from $44 \%$ at $t_{1}$ to $26 \%$ at $t_{3}$ in the PIT group and remaining essentially constant in the EMC group (39\% at both assessment points), there was no significant difference between the groups in the corresponding change in their PHQ-9 depression scores.

\section{Healthcare use}

Table 3 presents data on the participants' use of healthcare. Although the number of healthcare visits for primary care and specialists remained stable for both groups, we observed a reduction in antidepressant use immediately at $t_{2}$. At $t_{3}$ antidepressant use remained stable for the PIT group, whereas it increased for the EMC group. We also observed a significant difference in psychotherapy use; however, neither group frequently used psychotherapy at $t_{3}$ (PIT $n=3$; EMC $n=12$ ).

\section{Discussion}

This study suggests that clinically significant improvements can be achieved using a short-term intervention consisting of 12 PIT sessions in patients who experience chronic and disabling bodily symptoms that have no recognised treatment. ${ }^{8}$ In particular, this randomised controlled trial demonstrates that, in comparison 


\begin{tabular}{|c|c|c|c|c|}
\hline & $\begin{array}{c}\text { Psychodynamic } \\
\text { interpersonal therapy } \\
(n=107)\end{array}$ & $\begin{array}{c}\text { Enhanced medical } \\
\text { care } \\
(n=104)\end{array}$ & $t$ or $\chi^{2}$ & $P^{a}$ \\
\hline Age at entry, years: mean (s.d.) & $47.9(10.8)$ & $48.0(12.4)$ & $t=-0.05$ & 0.96 \\
\hline Female, $n(\%)$ & $67(63)$ & $72(69)$ & $\chi^{2}=1.45$ & 0.23 \\
\hline \multicolumn{5}{|l|}{ Education, $n(\%)$} \\
\hline$<10$ years & $48(45)$ & 37 (36) & \multirow{3}{*}{$\chi^{2}=1.85$} & \multirow{3}{*}{0.40} \\
\hline $10-12$ years & 39 (36) & 40 (39) & & \\
\hline $13+$ years & $21(20)$ & $26(25)$ & & \\
\hline Married, $n(\%)$ & $71(67)$ & $64(62)$ & $\chi^{2}=0.41$ & 0.52 \\
\hline \multicolumn{5}{|l|}{ Employment status, $n(\%)$} \\
\hline Employed & $44(41)$ & $44(42)$ & $\chi^{2}=0.09$ & 0.77 \\
\hline Retired & $27(25)$ & $18(17)$ & $\chi^{2}=1.78$ & 0.18 \\
\hline Other & $37(35)$ & $41(39)$ & $\chi^{2}=0.70$ & 0.40 \\
\hline \multicolumn{5}{|l|}{ Symptoms } \\
\hline Number of SCID somatoform symptoms (actual), mean (s.d.) & $10.0(3.9)$ & $10.6(4.0)$ & $t=-1.01$ & 0.28 \\
\hline Symptom duration, years: mean (s.d.) & $10.4(5.5)$ & $10.8(5.5)$ & $t=-0.45$ & 0.66 \\
\hline
\end{tabular}

\begin{tabular}{|c|c|c|c|c|}
\hline & \multirow{2}{*}{$\begin{array}{l}\text { Psychodynamic interpersonal } \\
\text { psychotherapy }(n=107) \\
\text { Mean (s.d.) }\end{array}$} & \multirow{2}{*}{$\begin{array}{l}\text { Enhanced medical care } \\
\qquad(n=104) \\
\text { Mean (s.d.) }\end{array}$} & \multicolumn{2}{|c|}{ Group difference } \\
\hline & & & Mean $(95 \% \mathrm{Cl})^{\mathrm{a}}$ & $P^{\mathrm{b}}$ \\
\hline \multicolumn{5}{|l|}{ Primary outcome } \\
\hline \multicolumn{5}{|c|}{ SF-36 Health Survey PCS } \\
\hline Baseline & $29.0(6.4)$ & $29.6(7.0)$ & $-0.6(-2.43$ to 1.21$)$ & 0.51 \\
\hline End of therapy & $31.3(8.1)$ & $32.6(11.0)$ & $-1.3(-3.90$ to 1.37$)$ & 0.70 \\
\hline Follow-up & $34.3(9.3)$ & $31.8(9.5)$ & 2.5 (0.16 to 5.09$)$ & 0.001 \\
\hline \multicolumn{5}{|l|}{ secondary outcomes } \\
\hline \multicolumn{5}{|c|}{ SF-36 Health Survey MCS } \\
\hline Baseline & $36.5(12.6)$ & $36.0(12.4)$ & $0.6(-2.85$ to 3.94$)$ & 0.75 \\
\hline End of therapy & $41.9(12.0)$ & $38.3(13.2)$ & $3.6(0.20$ to 7.06$)$ & 0.08 \\
\hline Follow-up & 40.1 (13.3) & $38.9(12.7)$ & $1.1(-2.25$ to 4.55$)$ & 0.73 \\
\hline \multicolumn{5}{|c|}{ PHQ Somatisation score } \\
\hline Baseline & $15.2(5.2)$ & $14.8(4.7)$ & $0.4(-0.94$ to 1.77$)$ & 0.48 \\
\hline End of therapy & $13.8(5.3)$ & $14.0(5.3)$ & $-0.2(-1.72$ to 1.14$)$ & 0.29 \\
\hline Follow-up & $12.7(5.8)$ & $13.8(5.3)$ & $-1.12(-2.65$ to 0.31$)$ & 0.01 \\
\hline \multicolumn{5}{|c|}{ PHQ Depression score } \\
\hline Baseline & $12.9(5.7)$ & $12.4(5.7)$ & $0.5(-1.03$ to 2.06$)$ & 0.47 \\
\hline End of therapy & $10.3(6.0)$ & $11.1(6.2)$ & $-0.8(-2.57$ to 0.84$)$ & 0.10 \\
\hline Follow-up & $10.5(6.0)$ & $11.2(6.5)$ & $-0.8(-2.46$ to 0.90$)$ & 0.08 \\
\hline \multicolumn{5}{|c|}{ Health Anxiety/Whiteley Index (WI-7) } \\
\hline Baseline & $3.9(2.1)$ & $4.1(2.0)$ & $-0.2(-0.79$ to 0.34$)$ & 0.42 \\
\hline End of therapy & $3.5(2.3)$ & $3.5(2.2)$ & $0.0(-0.62$ to 0.63$)$ & 0.52 \\
\hline Follow-up & $3.1(2.3)$ & $3.4(2.3)$ & $-0.3(-0.88$ to 0.31$)$ & 0.62 \\
\hline
\end{tabular}

with EMC, brief PIT provides greater improvement in physical quality of life at follow-up for those with multiple chronic and disabling medically unexplained symptoms and a high rate of comorbid depression and anxiety. ${ }^{27}$

Although a few controlled trials have evaluated the merits of CBT in patients with multiple somatoform symptoms, ${ }^{8,27}$ to our knowledge this is the first randomised controlled trial to demonstrate the efficacy of PIT in the same population. With a between-group effect size of 0.42 , PIT provided a moderate benefit to patients, ${ }^{31}$ which is consistent with the reported treatment effects of CBT in patients with bodily distress. ${ }^{32}$ We could demonstrate this effect even though interpersonal and in particular psychodynamic techniques usually employ more therapeutic sessions than used in our PIT intervention. Furthermore, the efficacy of PIT might be underestimated in an unselected group of patients following a randomisation procedure. $^{33}$

Kleinstaeuber et al recently meta-analysed 27 trials mainly applying cognitive-behavioural approaches, behavioural medical interventions or reattribution for multiple medically unexplained symptoms. ${ }^{27}$ The effects of these studies also proved to be small to modest for the majority of approaches and observable primarily for changes in physical symptoms ( $d=0.40$ at 1 -year follow-up) and - with reservations - for somatoform symptoms and 


\begin{tabular}{|c|c|c|c|c|}
\hline & $\begin{array}{l}\text { Psychodynamic interpersonal } \\
\text { psychotherapy }(n=107)\end{array}$ & $\begin{array}{l}\text { Enhanced medical care } \\
\qquad(n=104)\end{array}$ & Comparison & $P$ \\
\hline \multicolumn{5}{|c|}{ Number of healthcare visits, mean (s.d.) } \\
\hline \multicolumn{5}{|c|}{ Primary care } \\
\hline Baseline & $3.2(3.3)$ & $3.9(3.6)$ & & \\
\hline End of therapy & $3.9(5.1)$ & $3.4(3.8)$ & $0.22^{\mathrm{a}}$ & 0.68 \\
\hline Follow-up & $2.5(2.6)$ & $3.5(3.6)$ & $0.75^{\mathrm{a}}$ & 0.39 \\
\hline \multicolumn{5}{|c|}{ Specialist consultation } \\
\hline Baseline & $7.1(6.0)$ & $6.9(7.6)$ & & \\
\hline End of therapy & $7.4(7.4)$ & $5.3(6.6)$ & $3.25^{\mathrm{a}}$ & 0.11 \\
\hline Follow-up & $7.4(8.8)$ & $7.5(7.2)$ & $0.15^{\mathrm{a}}$ & 0.73 \\
\hline \multicolumn{5}{|c|}{ Concurrent antidepressant medication, $n$ (\%) } \\
\hline Baseline & $52(49)$ & $46(44)$ & & \\
\hline End of therapy & $41(38)$ & $44(42)$ & $3.57^{\mathrm{b}}$ & 0.17 \\
\hline Follow-up & $39(36)$ & $48(46)$ & $6.24^{\mathrm{b}}$ & 0.04 \\
\hline \multicolumn{5}{|c|}{ Psychotherapy at follow-up, $n$ (\%) } \\
\hline End of therapy & $6(6)$ & $6(6)$ & & \\
\hline Follow-up ${ }^{c}$ & $3(3)$ & $12(12)$ & $-1.98^{\mathrm{d}}$ & 0.05 \\
\hline \multicolumn{5}{|c|}{$\begin{array}{l}\text { a. Mixed models, between-group comparison of differences between baseline }\left(t_{1}\right) \text { and end of therapy }\left(t_{2}\right) \text {, and between } t_{1} \text { and 9-month follow-up }\left(t_{3}\right) \text { respectively, controlling } \\
\text { for initial frequency of visits and study site. } \\
\text { b. Chi-squared test. } \\
\text { c. No other psychotherapy allowed during treatment. } \\
\text { d. Mann-Whitney U-test. }\end{array}$} \\
\hline
\end{tabular}

healthcare use ( $d=0.33$ for each dimension). Another metaanalysis of psychodynamic psychotherapy for somatic symptom disorders demonstrated medium to high short-term and longterm effects on depression, anxiety and somatic symptoms. ${ }^{8}$ Somatic symptom disorders refer to chronic pain (nearly half of the studies), irritable bowel syndrome and a number of organically explained diseases. The authors stated that - despite the magnitude of the effects - the results were limited by the variable methodological quality of the identified studies and the heterogeneity in the analyses. ${ }^{8}$

Participants in our study who received EMC reported small improvements in health-related quality of life, with a withingroup effect size of 0.30 . This improvement is considerably greater than the control effect in similar psychotherapy trials (a mean control group effect size of 0.13 was observed in data collected from Abbass et al $)^{9}$ and thus contributes to the between-group effect size being moderate as reported here. We could not demonstrate a significant improvement in depression for PIT compared with EMC. This finding suggests that the assumption that an improvement in bodily function is solely or primarily related to an improvement in depression is incorrect. Kroenke et al described the same independence between bodily distress and depression: the majority of CBT studies improved patients' symptom severity but not their psychological distress. ${ }^{7,9}$

With the Bonferroni correction the reductions in the use of psychotherapy and antidepressants in the PIT group were not significant. This finding is not surprising, as we did not design this study to demonstrate such an effect. Moreover, the data regarding use of such therapies could potentially have been biased by the fact that neither the study personnel nor the patients had been masked to randomisation, and recommendations regarding future therapeutic procedures had been a part of the protocol. However, these data indicate that the observed improvement in physical quality of life for the PIT group cannot be explained by an increase in healthcare use. This conclusion recognises that the differential effect between the PIT and EMC groups became apparent only after the end of treatment. This result may signify that one important effect of the experimental intervention was an increase in specific interpersonal and health-related self-efficacy (i.e. the ability to care for one's own health in the context of others, both in and outside of the healthcare system). Previous studies have described comparable trajectories of change, including post-treatment improvements in psychotherapy, especially in PIT. ${ }^{9}$

We designed and carried out our study as an explanatory trial (with regard to the control of related conditions, the selection of the sample by using a widely used structured clinical interview, the delivery of standardised therapy and the strict allocation to the respective treatment condition). However, some features could allow it to be considered also as a pragmatic trial: ${ }^{34}$ we intended to examine the effect of PIT closely linked to routine clinical practice, with a group of patients characterised by some degree of heterogeneity, and we selected outcomes that represented a broad range of health gains.

\section{Strengths and limitations}

Strengths of the study include the rigorously controlled and monitored trial, its multisite setting, the relatively large sample size and moderate withdrawal rate, and the thorough joint pretrial development of its manual among all principal investigators. Our intention was to address a population of at least moderately impaired patients with multiple medically unexplained bodily symptoms without confinement to a particular functional syndrome, presenting at somatic out-patient departments. For this purpose we chose the diagnostic category of multisomatoform disorder, which has been used occasionally in other trials, such as that by Dickinson et al. ${ }^{35}$ The advantage of using this diagnostic construct as a common point of reference across single functional somatic symptoms and syndromes lies in its clinical relevance and its advanced integrative approach. ${ }^{6}$

Several limitations of our study, which are mainly related to the setting of the trial, the selection of patients and the comparability of the control intervention, should be discussed. The selection of the participating centres was not random, and the willingness to cooperate may have biased the selection. Accordingly the sample of out-patient departments cannot - in a strict sense - be deemed to be representative of multisomatoform disorders as a whole, as certain forms of medically unexplained bodily symptoms are likely to be treated in disciplines not represented in our study. The inclusion of patients with pain as 
the presenting symptom limits our ability to generalise these results to other somatoform disorders - although pain is by far the most frequent type of symptom in these disorders.

Finally, another limitation concerns the comparability of the two interventions: we are unable to determine whether the observed treatment effects in the experimental and control groups were due to the treatment itself or to non-specific effects related to the different amount of time spent with patients. Sumathipala et al demonstrated that structured care is as effective as CBT in patients with less severe somatic symptoms, both therapies consisting of six sessions of $30 \mathrm{~min}$ each. ${ }^{36}$ However, two arguments speak against this possibility: first, our differential treatment effect became apparent only after $t_{2}$; and second, in the PIT group the treatment effect was positively associated with the extent and intensity of interpersonal elements as measured with the adherence rating scale. ${ }^{37}$ We considered using a waiting-list condition to represent the natural course of the disease as an alternative to our control group. The decision to offer EMC was made for reasons that were both ethical (patients should receive the best available care, and EMC follows valid evidence-based guidelines) ${ }^{38}$ and practical (to ensure study participation).

\section{Future research}

Three immediate questions arise from the results of this study. First, does PIT produce improvements in the quality of life of patients with multisomatoform disorder comparable with those provided by CBT? Second - and perhaps of greater concern to health service planners - how does PIT perform compared with the care that patients are likely to receive in a real-world setting? ${ }^{38}$ Third, given that two studies have already shown that PIT - unlike other approaches such as reattribution ${ }^{39}$ - is one of few psychological interventions to demonstrate real cost savings, ${ }^{26,40}$ can we demonstrate such an effect for our study as well? So cost-effectiveness and cost-utility analyses for PIT compared with EMC in patients with multisomatoform disorder should be considered.

\section{Implications of the study}

Patients with severely disabling and chronic bodily distress who seek help from different somatic out-patient departments can be helped using PIT. This therapy also allowed patients to increase their physical functioning after the intervention. Enhanced medical care also provides relief for severely ill patients, but its effects are not of comparable sustainability. We need more information about this intervention's long-term sustainability and differential effects (i.e. those related to gender, individual psychological disease models and physiological data). Furthermore, we need to evaluate the benefits of this therapy in relationship to its potential cost savings. Knowing this information would enable us to better tailor our intervention and to increase its impact.

\section{Funding}

The German Research Foundation (DFG; He 3200/4-1) funded this study.

\section{Acknowledgements}

We thank those who participated in the study, the study therapists not involved in the preparation of the manuscript (Angela Angelowski, Claudia Denz, MD, Gerhard Haeckl, MD, Johanna Kahl, PhD, Meike Moritz, Eva Neumann, PhD, and Corinna van Niekerk, $M D$, Johanna Kahl, PhD, Meike Moritz, Eva Neumann, PhD, and Corinna van Niekerk,
$M D$ ) and the administrative staff in the participating out-patient departments who assisted $\mathrm{MD})$ and the admir
with this study.
Heribert Sattel, DiplPsych, Department of Psychosomatic Medicine and Psychotherapy, Klinikum Rechts der Isar, Technische Universität München, Claas Lahmann, MD, Department of Psychosomatic Medicine and Psychotherapy, Klinikum Rechts der Isar, Technische Universität München, and Department of Psychosomatic Medicine, University of Regensburg, Regensburg; Harald Gündel, MD, Department of Psychosomatic Medicine and Psychotherapy, Hannover Medical School and UIm University, Germany; Elspeth Guthrie, MD, Department of Psychiatry, University of Manchester, Manchester, UK; Johannes Kruse, MD, Department of Psychosomatic Medicine, University of Düsseldorf, and Centre for Psychosomatic Medicine, Justus Liebig University of Giessen; Michael Noll-Hussong, MD, Department of Psychosomatic Medicine and Psychotherapy, UIm University; Christian Ohmann, PhD, Coordination Centre for Clinical Trials, University of Düsseldorf; Joram Ronel, MD, Department of Psychosomatic Medicine and Psychotherapy, Klinikum Rechts der Isar, Technische Universität München; Martin Sack, PD, Department of Psychosomatic Medicine and Psychotherapy, Klinikum Rechts der Isar, Technische Universität München; Nina Sauer, MD, Department of Psychosomatic Medicine and Psychotherapy, University Hospital Heidelberg, and Department of Psychosomatic Medicine, Social Welfare Hospital, Hannover: Gudrun Schneider, MD, Department of Psychosomatic Medicine, University Hospita of Münster; Peter Henningsen, MD, Department of Psychosomatic Medicine and Psychotherapy, Klinikum Rechts der Isar, Technische Universität München, Munich, Germany

Correspondence: Prof. Dr med. Peter Henningsen, Department of Psychosomatic Medicine and Psychotherapy, Klinikum rechts der Isar, Technische Universität München,Langerstr. 3, D-81675 München, Germany. Email: p.henningsen@tum.de

First received 23 Feb 2011, final revision 17 Jun 2011, accepted 15 Sep 2011

\section{References}

1 Barsky AJ, Orav EJ, Bates DW. Somatization increases medical utilization and costs independent of psychiatric and medical comorbidity. Arch Gen Psychiatry 2005; 62: 903-10.

2 Henningsen $P$, Zipfel S, Herzog W. Management of functional somatic syndromes. Lancet 2007; 369: 946-55.

3 Kroenke K, Sharpe M, Sykes R. Revising the classification of somatoform disorders: key questions and preliminary recommendations. Psychosomatics 2007; 48: 277-85.

4 Creed F, Fink P, Guthrie E, Henningsen P, Rief W, Sharpe $M$, et al. Is there a better term than 'medically unexplained symptoms'? J Psychosom Res 2010; 68: $5-8$.

5 American Psychiatric Association. Somatoform Disorders - American Psychiatric Association DSM-5 Development. APA, 2010 (http:// www.dsm5.org/ProposedRevisions/Pages/SomatoformDisorders.aspx).

6 Fink P, Schröder A. One single diagnosis, bodily distress syndrome, succeeded to capture 10 diagnostic categories of functional somatic syndrome and somatoform disorders. J Psychosom Res 2010; 68: 415-25.

7 Kroenke K. Patients presenting with somatic complaints: epidemiology, psychiatric comorbidity and management. Int J Methods Psychiatr Res 2003; 12: $34-43$.

8 Kroenke K, Swindle R. Cognitive-behavioral therapy for somatization and symptom syndromes: a critical review of controlled clinical trials. Psychother Psychosom 2000; 69: 205-15.

9 Abbass A, Kisely S, Kroenke K. Short-term psychodynamic psychotherapy for somatic disorders: systematic review and meta-analysis of clinical trials. Psychother Psychosom 2009; 78: 265-74.

10 Kroenke K. Efficacy of treatment for somatoform disorders: a review of randomized controlled trials. Psychosom Med 2007; 69: 881-8.

11 Hardy G, Barkham M, Shapiro D, Guthrie E, Margison F (eds). PsychodynamicInterpersonal Psychotherapy. Sage, 2011 (in press).

12 Kroenke K, Spitzer RL, deGruy FV, Hahn SR, Linzer M, Williams JB, et al. Multisomatoform disorder: an alternative to undifferentiated somatoform disorder for the somatizing patient in primary care. Arch Gen Psychiatry 1997; 54: 352-8.

13 Spitzer RL, Kroenke K, Williams JB. Validation and utility of a self-report version of PRIME-MD: the PHQ primary care study. JAMA 1999; 282: 173744.

14 Ware JE, Sherbourne CD. The MOS 36-item Short-Form Health Survey (SF-36): I. Conceptual framework and item selection. Med Care 1992; 30: 473-83.

15 First MB, Spitzer RL, Gibbon M, Williams JBW. Structured Clinical Interview for DSM-IV-TR Axis I Disorders. Biometrics Research, New York State Psychiatric Institute, 2002.

16 Folstein MF, Folstein SE, McHugh PR. 'Mini-mental state'. A practical method for grading the cognitive state of patients for the clinician. J Psychiatr Res 1975; 12: 189-98. 
17 Seivewright H, Green J, Salkovskis P, Barrett B, Nur U, Tyrer P. Cognitivebehavioural therapy for health anxiety in a genitourinary medicine clinic: randomised controlled trial. Br J Psychiatry 2008; 193: 332-7.

18 Rudolf G, Henningsen P. Psychotherapeutic treatment of somatoform disorders [in German]. Z Psychosom Med Psychother 2003; 49: 3-19.

19 Guthrie E. Adaptation of the psychodynamic-interpersonal model to work with medically unexplained symptoms. In Psychodynamic-Interpersonal Psychotherapy (eds G Hardy, M Barkham, D Shapiro, E Guthrie, F Margison). Sage, 2011 (in press).

20 Henningsen P, Working Group PISO. Psychodynamic-interpersonal therapy for somatoform disorders (PISO) - a manualized short-term psychotherapy [in German]. In Practice of Psychodynamic Psychotherapy [in German] (eds M Beutel, S Doering, F Leichsenring, G Reich). Hogrefe, 2011 (in press)

21 Rief W, Henningsen P. Somatoform disorders [in German]. In Practice of Psychotherapy [in German] (eds W Senf, M Broda). Thieme, 2005.

22 Shapiro DA, Startup M. Measuring therapist adherence in exploratory psychotherapy. Psychother Res 1992; 2: 193-203.

23 Henningsen $\mathrm{P}$, Hartkamp N, Loew T, Sack M, Scheidt C, Rudolf G. Somatoform Disorders. Guidelines and Source Book [in German]. Schattauer, 2002.

24 Riddle DL, Lee KT, Stratford PW. Use of SF-36 and SF-12 health status measures: a quantitative comparison for groups versus individual patients. Med Care 2001; 39: 867-78.

25 Wiebe S, Matijevic S, Eliasziw M, Derry PA. Clinically important change in quality of life in epilepsy. J Neurol Neurosurg Psychiatry 2002; 73: 116-20.

26 Creed F, Fernandes L, Guthrie E, Palmer S, Ratcliffe J, Read N, et al. The cost-effectiveness of psychotherapy and paroxetine for severe irritable bowe syndrome. Gastroenterology 2003; 124: 303-17.

27 Kleinstaeuber M, Witthoeft M, Hiller W. Efficacy of short-term psychotherapy for multiple medically unexplained physical symptoms: a meta-analysis. Clin Psychol Rev 2011; 31: 146-60.

28 Pilowsky I. Dimensions of hypochondriasis. Br J Psychiatry 1967; 113: 89-93.
29 Fink $\mathrm{P}$, Ewald $\mathrm{H}$, Jensen J, Sørensen $\mathrm{L}$, Engberg $\mathrm{M}$, Holm $\mathrm{M}$, et al. Screening for somatization and hypochondriasis in primary care and neurological in-patients: a seven-item scale for hypochondriasis and somatization. J Psychosom Res 1999; 46: 261-73.

30 Altman DG, Schulz KF, Moher D, Egger M, Davidoff F, Elbourne D, et al. The revised CONSORT statement for reporting randomized trials: explanation and elaboration. Ann Intern Med 2001; 134: 663-94.

31 Cohen J. Statistical Power Analysis for the Behavioral Sciences (2nd edn). Erlbaum, 1988.

32 Looper KJ, Kirmayer LJ. Behavioral medicine approaches to somatoform disorders. J Consult Clin Psychol 2002; 70: 810-27.

33 Fonagy P. Psychotherapy research: do we know what works for whom? Br J Psychiatry 2010; 197: 83-5.

34 Roland M, Torgerson DJ. What are pragmatic trials? BMJ 1998; 316: 285

35 Dickinson WP, Dickinson LM, deGruy FV, Main DS, Candib LM, Rost K. A randomized clinical trial of a care. recommendation letter intervention for somatization in primary care. Ann Fam Med 2003; 1: 228-35.

36 Sumathipala A, Siribaddana S, Abeysingha MRN, De Silva P, Dewey M, Prince $M$, et al. Cognitive-behavioural therapy $v$. structured care for medically unexplained symptoms: randomised controlled trial. Br J Psychiatry 2008; 193: 51-9.

37 Sattel H, Kittner A, Henningsen P. A brief psychodynamic-interpersonal psychotherapy for patients with multisomatoform disorder (PISO) one size fits all? Psychother Psych Med 2011; 61: 111.

38 Krause MS, Lutz W. What should be used for baselines against which to compare treatments' effectiveness? Psychother Res 2009; 19: 358-67.

39 Morriss R, Dowrick C, Salmon P, Peters S, Dunn G, Rogers A, et al. Cluster randomised controlled trial of training practices in reattribution for medically unexplained symptoms. Br J Psychiatry 2007; 191: 536-42.

40 Guthrie E, Moorey J, Margison F, Barker H, Palmer S, McGrath G, et al. Cost-effectiveness of brief psychodynamic-interpersonal therapy in high utilizers of psychiatric services. Arch Gen Psychiatry 1999; 56: 519-26.

\section{DSM}

\section{Peter Tyrer}

DSM is an American classification system that has dominated since 1980. It is disliked by many for reducing diagnostic skills to a cold list of operational criteria, yet embraced by researchers believing that it represents the first whiff of sense in an area of primitive dogma. It has almost foundered by confusing reliability with validity but the authors seem to recognise its errors and are hoping for rebirth in its 5th revision due in May 2013. The initials do not stand for Diagnosis as a Source of Money or Diagnosis for Simple Minds but the possibility of confusion is present. 